\section{OP0189 IDENTIFICATION OF NEW AND RARE VARIANTS IN ABCG2, SLC22A1 AND ALDH16A1 GENES IN CRYSTAL- PROVEN EARLY-ONSET GOUT}

C. Collet ${ }^{1}$, H. Morel ${ }^{1}$, M. Ricquebourg ${ }^{1}$, M. Cohen-Solal ${ }^{2}$, J.-L. Laplanche ${ }^{1}$, T. Pascartt ${ }^{3}$ T. Bardin ${ }^{4}$, F. Lioté ${ }^{4}$, P. Richette ${ }^{4}$, H.K. Ea ${ }^{2,4} .{ }^{1}$ Biochemistry, Hôpital Lariboisière; ${ }^{2}$ Bioscar, Inserm UMR 1132, Paris; ${ }^{3}$ rheumatology, Groupement des Hôpitaux de I'Institut Catholique de Lille, Lille; ${ }^{4}$ rheumatology, Hôpital Lariboisière, Paris, France

Background: Early-onset or juvenile gout (EOG) without hypoxanthine-guanine phosphoribosyltransferase enzyme deficiency (HPRT, OMIM 300323) and not related to familial juvenile hyperuricemic nephropathy (UMOD, OMIM 300323) is a rare gout phenotype characterised by a first flare in adolescence or in young adulthood. While numerous genome wide association studies (GWAS) have been done in classical and late-onset gout, very few studies have been performed in EOG patients. Moreover, until now most genetic studies only assess association between pre-defined single nucleotide polymorphisms (SNP) and gout.

Objectives: Our aim was to identify the genetic variants of clinically confirmed EOG by screening all exons of gout-associated genes with targeted Next-Generation Sequencing (NGS) approach.

Methods: Twenty-six urate crystal-proven gout patients with first flare occurring before the age of 30 years were included. Gout history, comorbidities and patient characteristics were recorded. All participants provided written informed consent to genetic analysis. After DNA extraction from total blood samples, the NGS libraries were prepared with surselect QXT (Agilent) and sequencing was performed with miseq (Illumina).

The multigene panel included 80 genes described in GWAS and genes involved in rare diseases such as HPRT and UMOD.

Results: Twenty-six patients (24 men, 20 Caucasians, 5 Asians and 1 African) with crystal-proven gout had experienced their first flare at a mean age of 22.8 years [14-29] Gout duration was 11.5 years [1-46] and clinical tophi observed in 9 patients. Mean age was 37.5 [24-69] years and mean body mass index $27.6 \mathrm{~kg} /$ m2 [20.1-40.7]. Ten patients were overweight, 5 had obesity, 1 hypertension, 0 diabetes mellitus, 7 dyslipidemia and 10 chronic kidney disease stages 2-4. Mean serum urate level was $527 \mu \mathrm{mol} / \mathrm{L}$ [270-803]. Amongst 26 affected patients, 7 had a molecular anomaly (26.9\%). Six patients harboured one rare or novel variant in ABCG2 (three Caucasian patients), ALDH16A1 (two Caucasian patients) and SLC22A11 (one African patient). Two other patients (one Caucasian and one Asian) carried an association of variants in both ABCG2 and ALDH16A1. All variants had a Minor Allele Frequency (MAF) below $0.3 \%$ or were never described in public databases. All variant were considered as probably pathogenic according to in silico predictive algorithms. Interestingly, the well-known p.Gln141Lys SNP of $A B C G 2$ was identified in 3 Asian patients (11.5\%) at homozygous level.

Conclusions: Our finding of very rare and novel pathogenic variants in ABCG2, $A L D 16 H 1$ and SLC22A11 genes provides better insights of the molecular pathogenesis in early-onset juvenile gout. However, our results also highlight the involvement of yet undetermined genes in this population.

Disclosure of Interest: None declared

DOI: 10.1136/annrheumdis-2018-eular.4981

THURSDAY, 14 JUNE 2018

\section{Treatments: friend or foe?}

\section{OP0190 THE ROLE OF NSAIDS IN THE ASSOCIATION BETWEEN OSTEOARTHRITIS AND CARDIOVASCULAR DISEASES: A POPULATION-BASED COHORT STUDY}

M. Atiquzzaman ${ }^{1}$, J. Kopec ${ }^{2}$, M.E. Karim², H. Wong ${ }^{2}$, A. Anis ${ }^{2} .{ }^{1}$ Faculty of Pharmaceutical Sciences; ${ }^{2}$ School of Population and Public Health, University of British Columbia, Vancouver, Canada

Background: Worldwide, osteoarthritis $(\mathrm{OA})$ is a major musculoskeletal disorder. Recent research suggests that $\mathrm{OA}$ is an independent risk factor for cardiovascular disease (CVD). ${ }^{1}$ The relationship is complicated because non-steroidal antiinflammatory drugs (NSAIDs), a proven risk factor for CVD, are frequently used for the treatment of OA. ${ }^{2}$ Researchers have hypothesised that NSAID use in the causal pathway between OA and CVD is what may ultimately impact these patients to develop CVD, this pathway has yet to be studied. ${ }^{3.4}$

Objectives: The objective of this study was to disentangle the role of NSAID in the increased risk of CVD among OA patients.

Methods: This longitudinal study was based on linked health administrative data from British Columbia, Canada. From a population-based cohort of 720,055 British Columbians, we matched on age and sex to assemble 7,743 OA patients and 23229 non-OA controls (1:3 ratio). We used multivariable Cox proportional hazards models to estimate the risk of developing incident CVD (primary outcome) as well as ischaemic heart disease (IHD), congestive heart failure (CHF) and stroke (secondary outcomes). To estimate the mediating effect of NSAIDs, defined as current use of NSAID using linked prescription dispensing records, in the OACVD relationship, we implemented a marginal structural model.

Results: People with OA had $23 \%$ higher risk of developing CVD compared to people without OA after adjusting for SES, BMI, hypertension, diabetes, hyperlipidemia, COPD, and Romano comorbidity score, adjusted $\mathrm{HR}(95 \% \mathrm{Cl})$ was 1.23 $(1.17,1.29)$. Adjusted HR (95\% Cl) was $1.42(1.33,1.52), 1.17(1.10,1.27), 1.14$ $(1.08,1.24)$ for $\mathrm{CHF}, \mathrm{IHD}$ and stroke, respectively. Approximately $67.51 \%$ of the total effect of OA on the increased risk of CVD was mediated through current NSAID use. Among the secondary outcomes, approximately $44.77 \%$ of increased $\mathrm{CHF}$ risk was mediated through current NSAID use. More than $90 \%$ of the total effects on IHD and stroke was mediated through the current NSAID use. Conclusions: Our study is the first to evaluate the mediating role of NSAID use in the OA-CVD relationship based on population-based health administrative data The results of this study also indicate that $O A$ is an independent risk factor for CVD. Our findings suggest that the mediating role of NSAID use substantially contributes to the OA-CVD association.

\section{REFERENCES}

[1] Wang H, Bai J, He B, Hu X, Liu D. Osteoarthritis and the risk of cardiovascular disease: A meta-analysis of observational studies. Sci Rep [Internet] 2016 Dec [cited 2017 Sep 6];6(1). Available from http://www.nature.com/ articles/srep39672

[2] Trelle S, Reichenbach S, Wandel S, Hildebrand P, Tschannen B, Villiger PM, et al. Cardiovascular safety of non-steroidal anti-inflammatory drugs: network meta-analysis. BMJ. 2011 Jan 11;342:c7086.

[3] Rahman MM, Kopec JA, Anis AH, Cibere J, Goldsmith CH. Risk of Cardiovascular Disease in Patients With Osteoarthritis: A Prospective Longitudinal Study: Cardiovascular Disease in Osteoarthritis Patients. Arthritis Care Res. 2013 Dec;65(12):1951-8.

[4] Link Between OA and CVD Risk [Internet]. arthritis.org. [cited 2017 Oct 23]. Available from: http://www.arthritis.org/living-with-arthritis/comorbidities/ heart-disease/osteoarthritis-ups-cvd-risk.php

Disclosure of Interest: None declared

DOI: 10.1136/annrheumdis-2018-eular.2640

\section{OP0191 ALL-CAUSE MORTALITY AND CARDIOVASCULAR DEATH IN HYDROXYCHLOROQUINE USERS IN RHEUMATOID ARTHRITIS PATIENTS - A POPULATION BASED DANISH COHORT STUDY}

K. Soeltoft ${ }^{1}$, J. Hallas ${ }^{2}$, M.C.M. Wasko ${ }^{3}$, A.B. Pedersen ${ }^{4}$, S.P. Ulrichsen ${ }^{4}$

T. Ellingsen ${ }^{1}{ }^{1}$ Dept. of Rheumatology; ${ }^{2}$ Dept of Clinical Chemistry and Pharmacology, Odense University Hospital, Odense, Denmark; ${ }^{3}$ West Penn Hospital, Pittsburgh, Pittsburgh, USA; ${ }^{4}$ Dept. of Clinical Epidemiology, Aarhus University Hospital, Aarhus, Denmark

Background: Rheumatoid arthritis $(\mathrm{RA})$ is associated with a marked increase in cardiovascular comorbidity and mortality..' The increased risk is present from the earliest stages of the disease and evidence suggests an overlap in the pathogenic features leading to $\mathrm{RA}$ and atherosclerosis.

Hydroxychloroquine (HCQ) is used to treat RA in combination with methotrexate and has been associated with decreased risk of type II diabetes and dyslipidaemia among RA patients. Also HCQ has improved survival rates when used to treat other inflammatory diseases, e.g. systemic lupus erythematosus.. 'The evidence regarding RA patients is scarce.

Objectives: We wish to examine whether HCQ would affect the incidence rates of cardiovascular diseases, type II diabetes, cardiac - and all-cause mortality among Danish RA patients in an observational cohort study.

Methods: We identified all incident RA patients during the period of 2004 through 2014 in Denmark. HCQ initiators were compared with non-users of HCQ, stratified on rheumatoid factor positivity. Each HCQ initiator was matched to a non-HCQ initiator by their propensity score (PS). In the PS all relevant available cardiovascular drugs and comorbidities were included. All together we had 3,742 RA patients in each group

Results: We found a significant reduction in all-cause mortality and cardiovascular related death among $\mathrm{HCQ}$ initiators, with a hazard ratio of 0.83 (95\% confidence interval $[\mathrm{Cl}] 0.71-0.97)$ and $0.78(95 \% \mathrm{Cl}: 0.61$ to 0.99$)$, respectively. We did not find any association between HCQ use and development of type II diabetes or specific ischaemic events (myocardial infarctions and ischaemic strokes). 


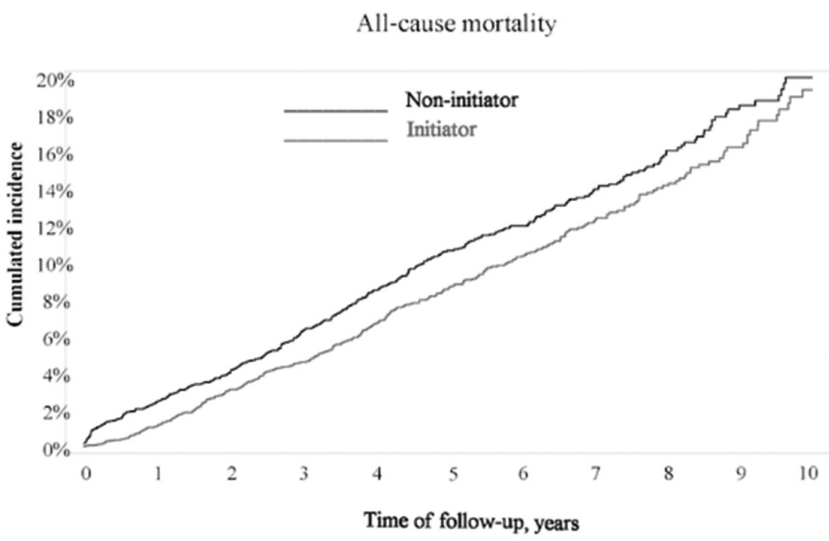

Conclusions: The beneficial properties on survival seen with HCQ use in other inflammatory diseases seem also applicable to RA patients. These results needs confirmation in a randomised setting but justifies the use of $\mathrm{HCQ}$ in combination with other conventional synthetic disease-modifying antirheumatic drugs even though the effect of $\mathrm{HCQ}$, per se, on RA disease activity is limited.

\section{REFERENCE:}

[1] Rempenault C, Combe B, Barnetche T, Gaujoux-Viala C, Lukas C, Morel $\mathrm{J}$, et al. Metabolic and cardiovascular benefits of hydroxychloroquine in patients with rheumatoid arthritis: A systematic review and meta-analysis. Ann Rheum Dis 2018 Jan;77(1):98-103.

Disclosure of Interest: None declared

DOI: 10.1136/annrheumdis-2018-eular.5770

\section{OP0192 METHOTREXATE USE AND THE RISK FOR CARDIOVASCULAR DISEASE AMONG RHEUMATOID PATIENTS INITIATING BIOLOGIC DISEASE-MODIFYING ANTIRHEUMATIC DRUGS}

F. Xie, L. Chen, H. Yun, E. Levitan, P. Muntner, J.R. Curtis. University of Alabama at Birmingham, Birmingham, USA

Background: Methotrexate (MTX) has been associated with reduced risk for CVD in several studies conducted among rheumatoid arthritis (RA) patients never exposed to biologic disease-modifying antirheumatic drugs (bDMARDS). Effect of concomitant MTX use on CVD risk among RA patients initiating bDMARDS remains unknown.

Objectives: The objective of this study was to assess the CVD risk associated with MTX use among RA patients initiate bDMARDS, overall, and by each bDMARDS initiated.

Methods: A retrospective cohort study was conducted using 2006-2015 Medicare claims data for RA patients. Follow up started at initiation (index date) and ended at earliest of 1) end of exposure of the specific bDMARDS agent (days of supply plus 90 days extension), 2) switched to other bDMARDS or tofacitinib, 3) CVD event, 4) death date, 5) loss of Medicare coverage, 6) end of study (September 30,2015$)$. MTX use was defined as 1) concomitant MTX use, with prescription for MTX within 120 days after index date and 2) time varying MTX, defined as prescription date to prescription date plus days of supply without extension. For sensitivity analysis, a 90 day extension was added to days of supply. The primary outcome was composite of incident MI, incident stroke and fatal CVD. Fatal CVD were identified by a claims based algorithm with PPV $\geq 80 \%$.

Incidence rates (IR) and 95\% confidence intervals $(\mathrm{Cl})$ were calculated using Poisson regression. Overall association between MTX use (versus no MTX) and risk of CVD were assessed using Cox regression. Given that the interactions between MTX and background bDMARDs was significant, we performed contrast (MTX Yes vs No) to examine the association between MTX and risk for CVD for each underlying bDMARDS in one model. A subgroup analysis limited the cohort to RA patients with previous exposure to MTX was conducted to ensure consistency of findings.

Results: A total of 88,255 DMARDS initiations (64 218 patients) were included in this study. The average age at initiation was $64.6(12.3)$ years, $84.0 \%$ were female, $68.2 \%$ were non-Hispanic white. The crude IRs for CVD were $13.1(95 \%$ $\mathrm{Cl}: 12.2$ to 14.0$)$ and 18.7 (95\% Cl: 17.6 to 19.9$)$ events per 1000 person years for RA patients with and without concomitant MTX respectively. The crude IRs for CVD were 12.1 (95\% Cl: 11.1 to 13.2$)$ and 17.9 (95\% Cl: 16.9 to 18.8$)$ events per 1000 person years for RA patients with and without time varying MTX respectively. IRs for individual bDMARDS are shown in figure. P-value for interaction between concomitant MTX and background bDMARDS was 0.0189 and p-value for interaction between time varying MTX and background bDMARDS was 0.0030 . The contrast HRs for concomitant MTX ranged from $0.61(0.37,1.01)$ for golimumab initiators to $0.97(0.74,1.26)$ for adalimumab initiators (figure 1). The contrast HRs for time varying MTX ranged from $0.58(0.35,0.96)$ for certolizumab initiators to $0.90(0.68,1.18)$ for adalimumab initiators.

Results were robust in sensitivity and subgroup analyses.

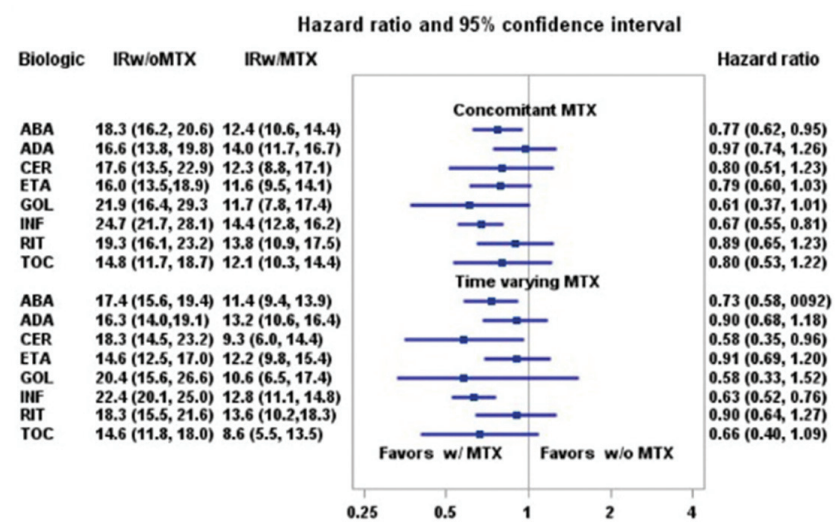

Conclusions: Our observational study suggests an overall $23 \%$ reduction of CVD risk associated with concomitant MTX use. The effect sizes vary among background bDMARDS.

Disclosure of Interest: F. Xie: None declared, L. Chen: None declared, H. Yun Grant/research support from: BMS, E. Levitan Grant/research support from: Amgen, Consultant for: Amgen, Novartis, P. Muntner: None declared, J. Curtis Grant/research support from: AbbVie, Amgen, BMS, Corrona, Janssen, Lilly, Myriad, Pfizer, Roche/Genentech, UCB, Consultant for: AbbVie, Amgen, BMS, Corrona, Janssen, Lilly, Myriad, Pfizer, Roche/Genentech, UCB DOI: 10.1136/annrheumdis-2018-eular.6796

\section{OP0193 TOCILIZUMAB AND THE RISK FOR CARDIOVASCULAR DISEASE EVENTS AMONG RHEUMATOID ARTHRITIS PATIENTS: A DIRECT COMPARISON IN REAL WORLD SETTING}

F. Xie, H. Yun, E. Levitan, P. Muntner, J.R. Curtis. University of Alabama at Birmingham, Birmingham, USA

Background: Multiple studies have observed unfavourable changes in lipid profile associated with tocilizumab (TCZ, anti-IL6 receptor antagonists) and some other rheumatoid arthritis (RA) therapies. The real-world cardiovascular disease (CVD) risk associated with the first anti IL-6R medication for RA, TCZ, remains uncertain.

Objectives: The objective of this study was to assess the CVD risk associated with TCZ compared to individual tumour necrosis inhibitor (TNFi) therapies, as well as to other biologics used for RA (e.g. rituximab, abatacept).

Methods: Using 2006-2015 Medicare and MarketScan claims data, we conducted a retrospective cohort study among RA patients who initiated biologic disease-modifying antirhematic drugs (bDMARDS) after January 1, 2010 and had at least 365 days medical and pharmacy coverage before initiation. The primary outcome was a composite of myocardial infarction (MI), stroke, and fatal CVD assessed using a validated method. Subgroups analyses were done for RA patients experienced to other bDMARDs before initiation and by stratifying patients with respect to key CVD risk factors to identify both higher and lower CVD risk patients. We provided descriptive statistical for each exposure group. Incidence rates and $95 \%$ confidence intervals were calculated using Poisson regres sion. COX regression was used to generate unadjusted and adjusted hazard ratio.

Results: We identified 354486 (Medicare 206,275+MarketScan 148,211) RA patients and 463446 (Medicare 271,832+MarketScan 191,614) initiations of bDMARDS. After applying inclusion and exclusion criteria, the final cohort contained 88463 (Medicare 46,648+MarketScan 41,815) RA patients and 117493 (Medicare 61,715+MarketScan 55,778) episodes. The mean (SD) age was 64.7 (12.1) in Medicare and 52.2 (12.3) in MarketScan. The majority of patients were female $(83.9 \%$ in Medicare and $80.5 \%$ in MarketScan), and $68.6 \%$ were non-Hispanic White in Medicare. TCZ users were similar to abatacept and rituximab users except that TCZ users were less likely to be naïve to bDMARDS. Compared to TNFi users, TCZ users were more likely to be white, with history of CVD (other than $\mathrm{MI}$ or stroke), heart failure, atrial fibrillation, hospitalisation and had more 